Supporting Information

\title{
Functionalized, Complementary Live-Charge Quantum Surface-Enhanced Raman Scattering Probes for Biomolecular Detection
}

\author{
Rupa Haldavnekar b, c, d, e, f, Dr. Krishnan Venkatakrishnan ${ }^{\text {a, c, d, e, }}$ Ph.D., \\ Dr. Bo Tan ${ }^{\text {a, e Ph.D. }}$
}

a. Affiliated Scientist, Keenan Research Center for Biomedical Science, St. Michael's Hospital, 30 Bond Street, Toronto, ON Canada M5B 1W8

b. Institute for Biomedical Engineering, Science and Technology (iBEST), Toronto, Canada

c. Ultrashort Laser Nanomanufacturing research facility, Department of Mechanical and Industrial Engineering, Ryerson University, 350 Victoria Street, Toronto, ON Canada M5B 2K3

d. BioNanoInterface Facility, Department of Mechanical and Industrial Engineering, Ryerson University, 350 Victoria Street, Toronto, ON Canada M5B 2K3

e. Nanocharacterization Laboratory, Department of Aerospace Engineering, Ryerson University, 350 Victoria Street, Toronto, ON Canada M5B 2K3

f. Department of Biomedical Engineering, Ryerson University, 350 Victoria Street, Toronto, ON Canada M5B 2K3

* Corresponding Author: venkat@ryerson.ca 416-979-5000 ext. 4984 


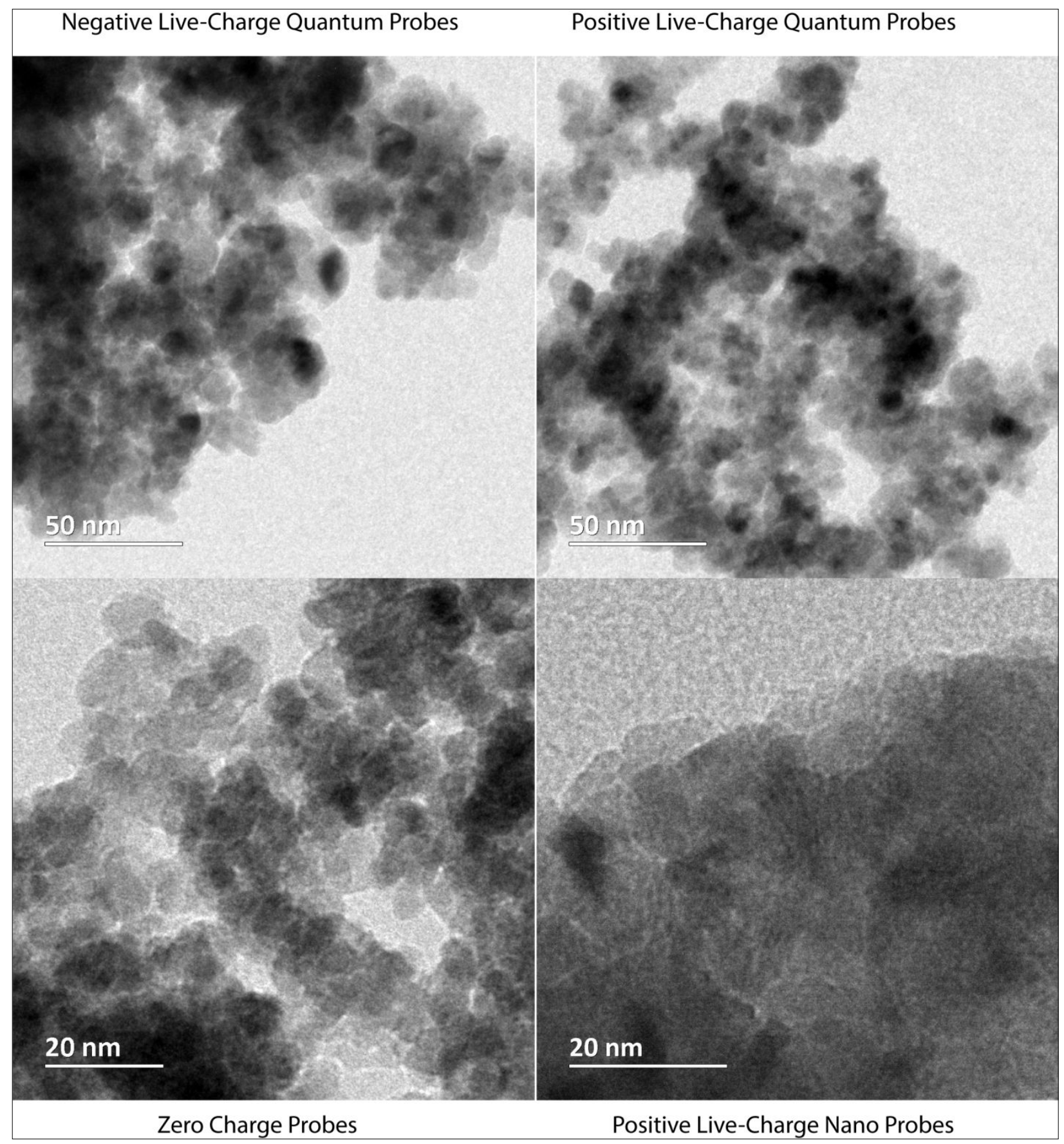

Supporting Fig S1- HRTEM of Positive Live-Charge, Zero Charge , Negative Live-Charge Quantum Probes and Positive Live-Charge Nano Probes 


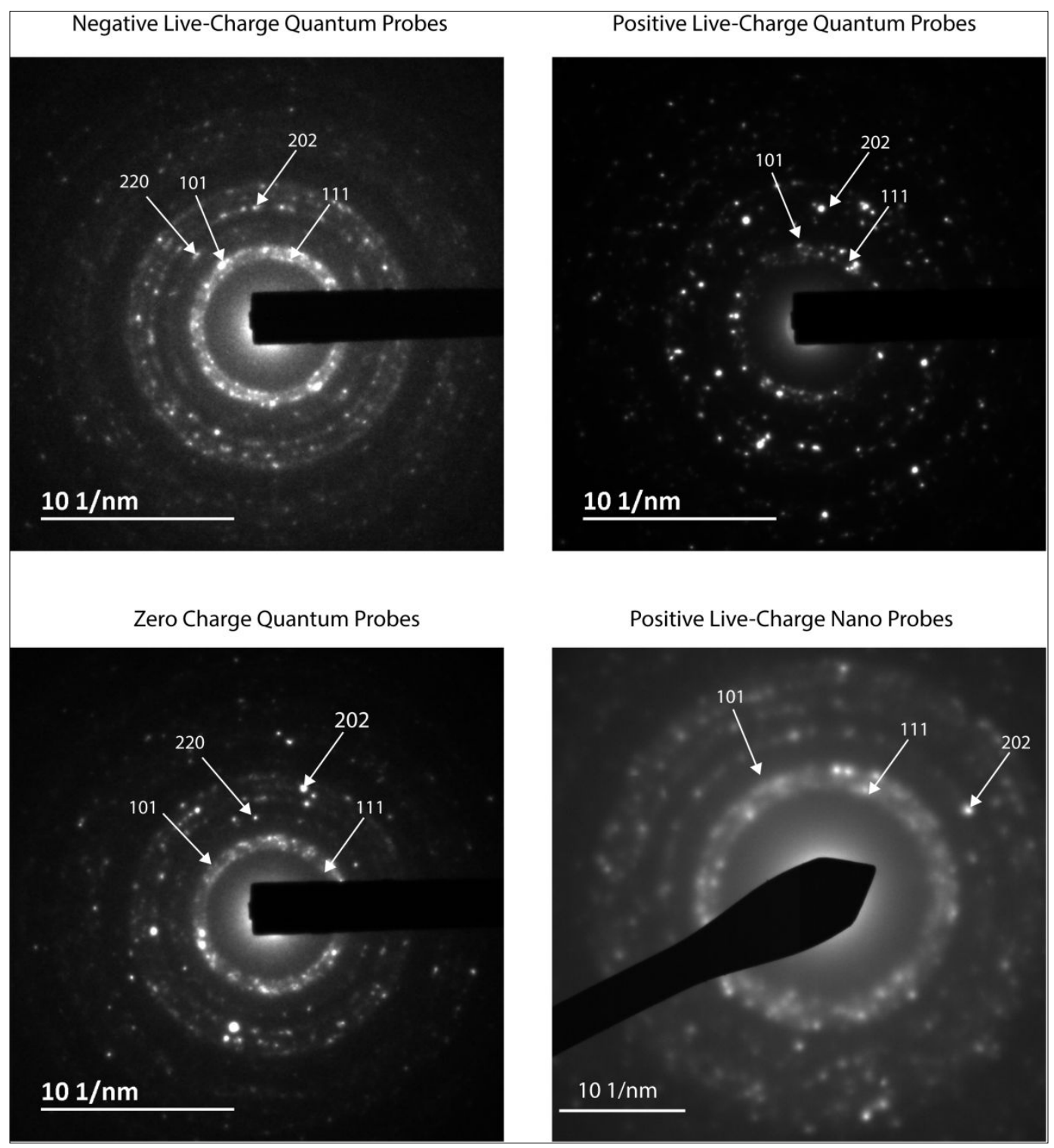

Supporting Fig S2- SAED of Live-Charge Probes demonstrating Polycrystalline Morphology \& Multi-planer Orientation 


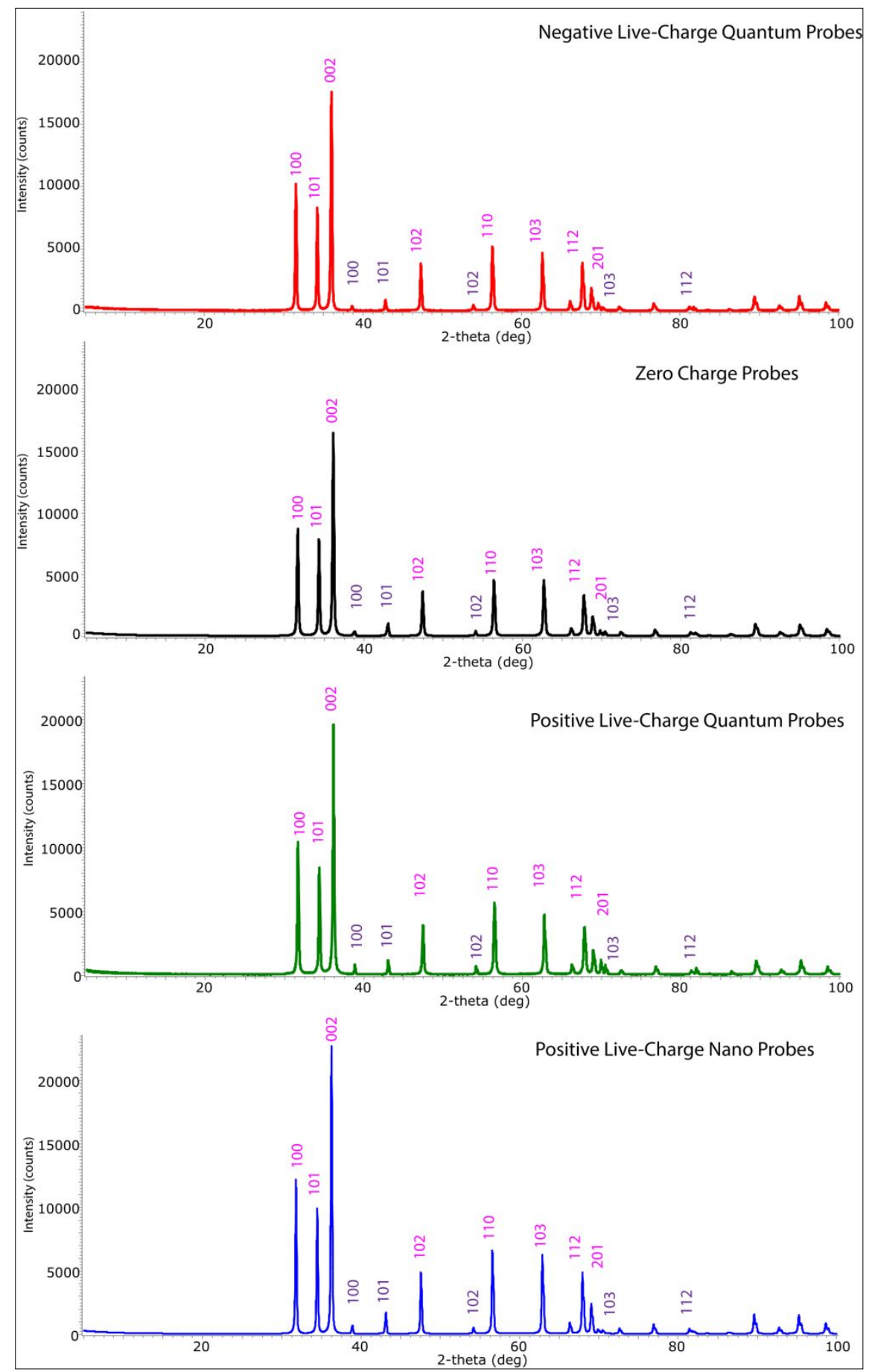

Supporting Fig S3- XRD Analysis of Live-Charge quantum probes. Planer orientation in pink represents Zinc Oxide and in purple represents zinc 


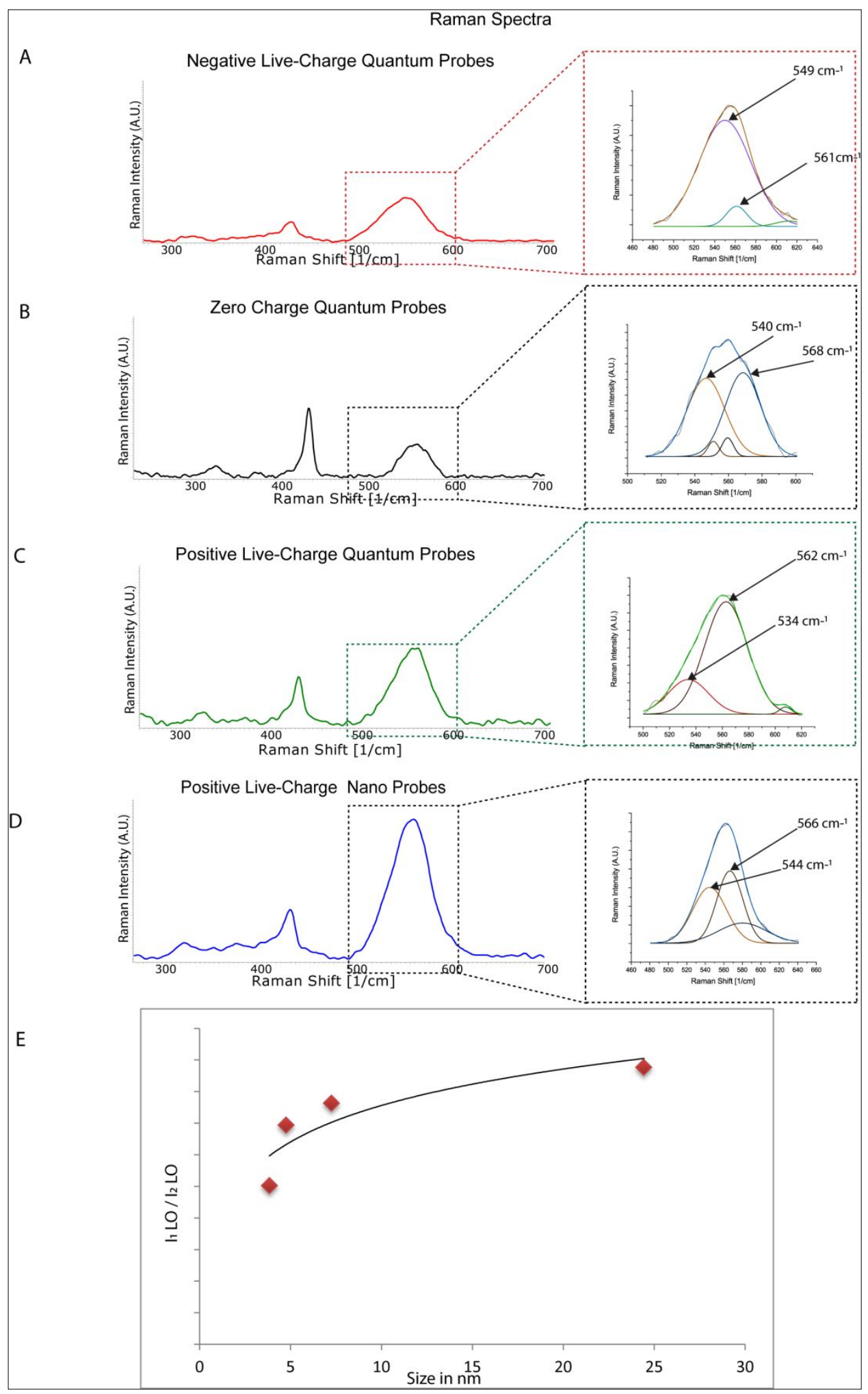

Supporting Fig S4- A-D: Raman Analysis of Live-Charge probes demonstrated reduced crystallinity and increased oxygen vacancies with induced charge , E: Ratio between second order and first order Raman scattering cross section as a function of probe size. 


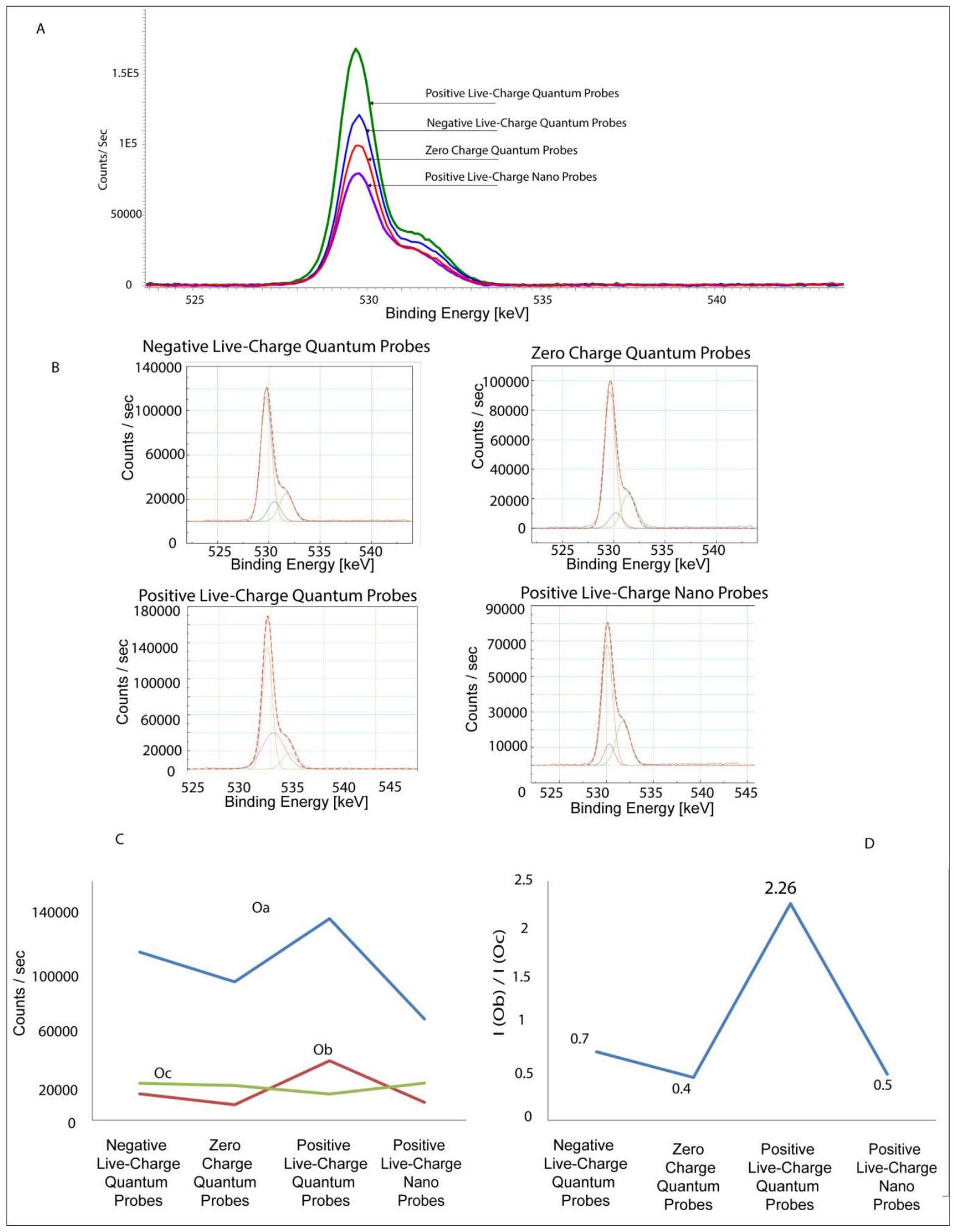

Supporting Fig S5- A: Comparison of XPS Oxygen Spectra for four types of probes B: XPS Oxygen Spectra deconvolution C: Comparison of the relative intensities of $\mathrm{Oa}$ ( $\mathrm{Zn}-\mathrm{O}$ bond and Wurtzite structure), Ob ( Oxygen deficient region) and Oc ( Chemisorbed or dissociated Oxygen) for four types of probes D: Comparison of Ratio of I (Ob)/ I (Oc) for four types of probes 


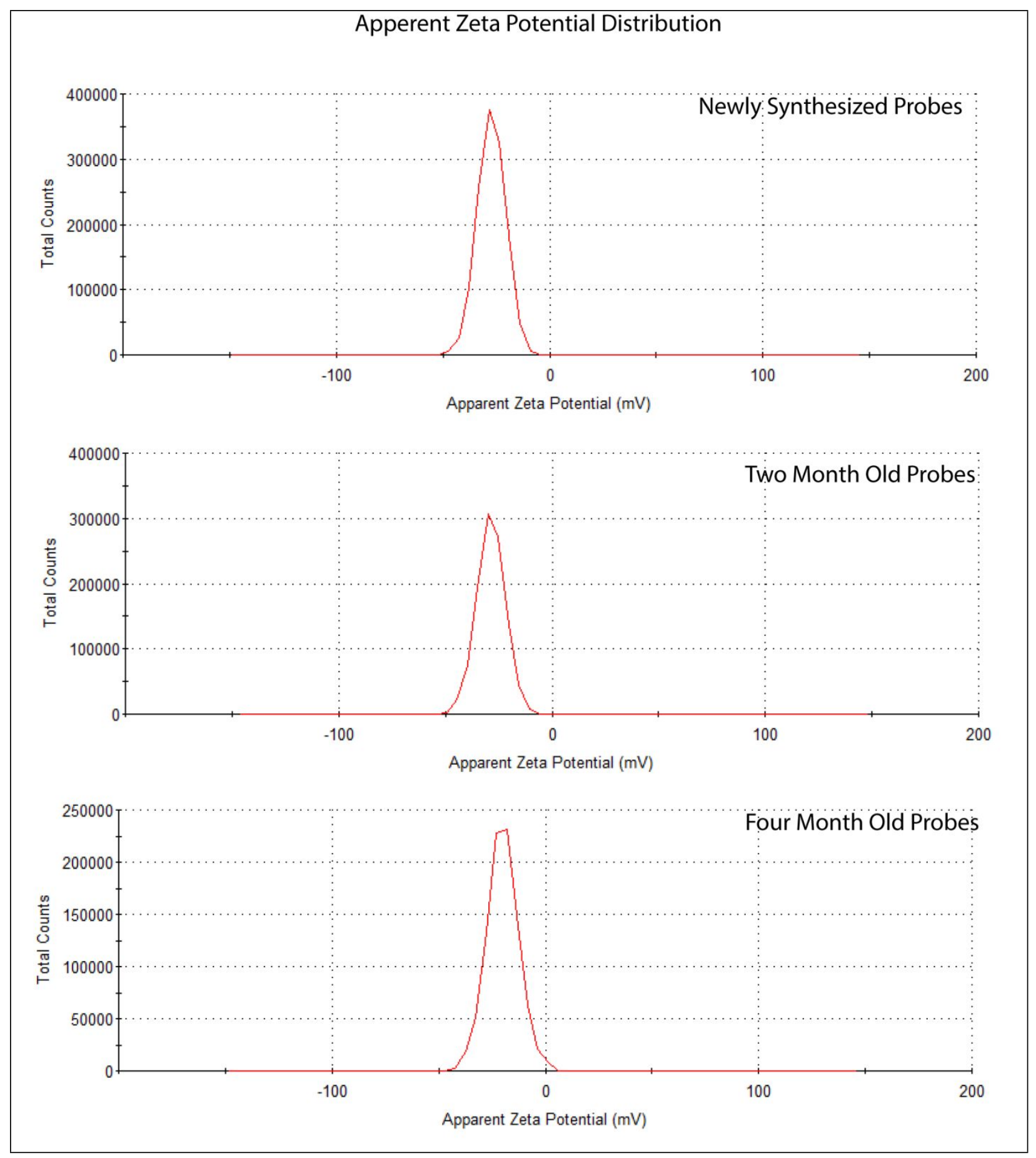

Supporting Fig S6- Stability of Live-Charge probes 


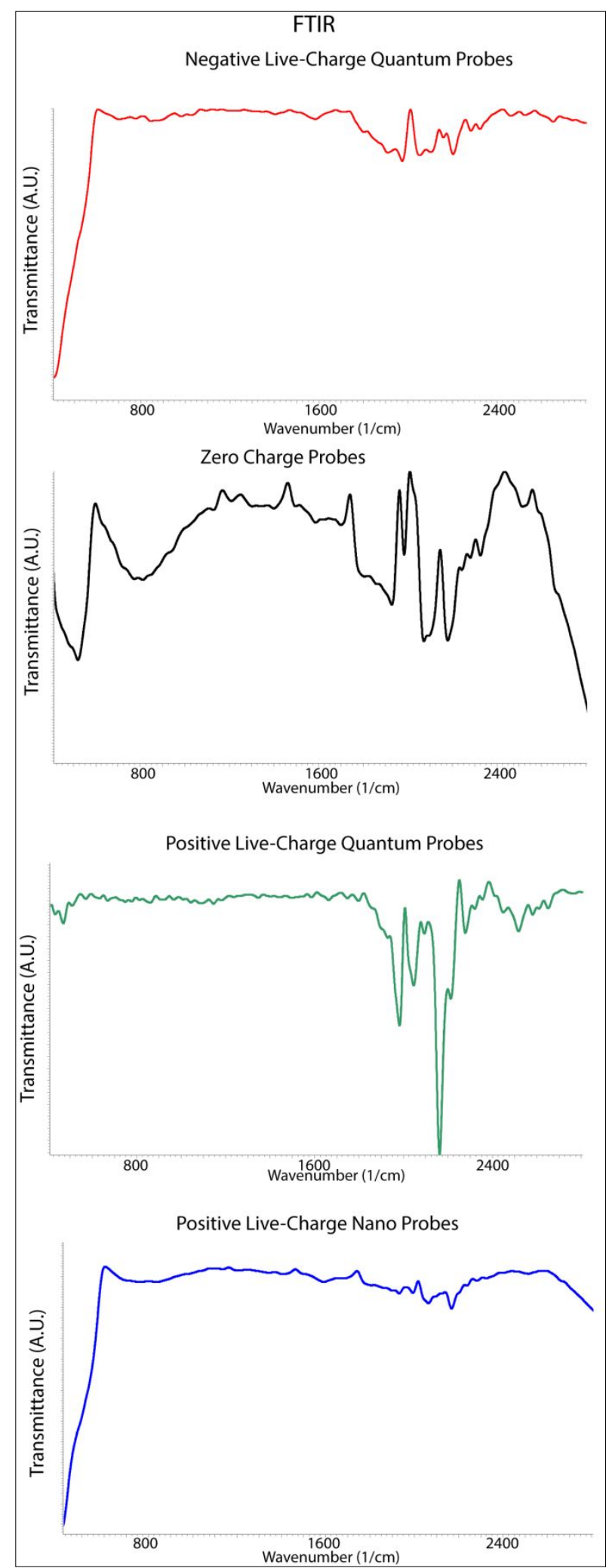

Supporting Fig S7: FTIR f Live-Charge Quantum Probes 

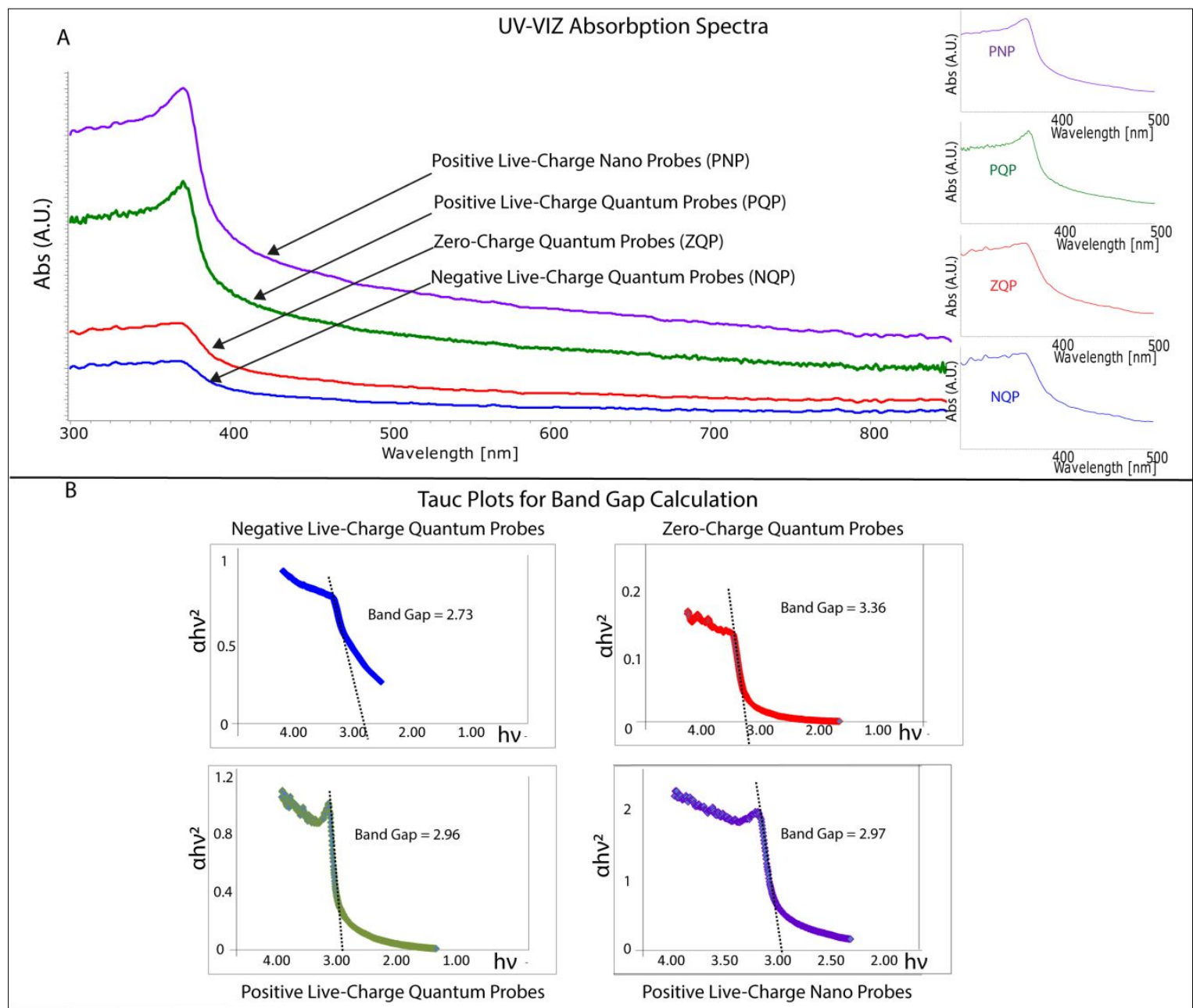

C

High Resolution Valence Band Edge XPS
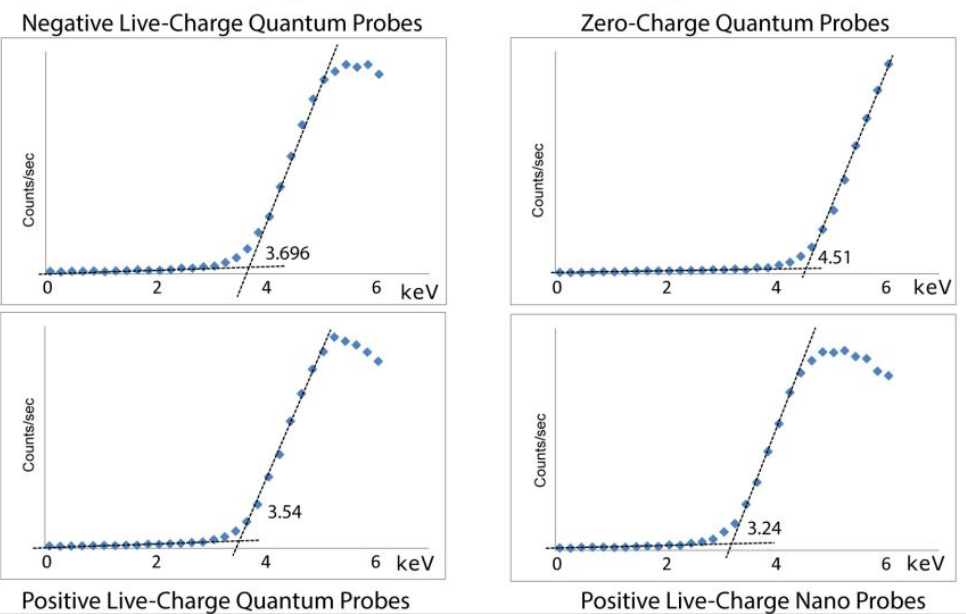

Supporting Fig S8: A. UV-VIZ absorption spectra, B. Tauc plots for calculation of band gap and C. High resolution Valence band XPS spectra for 1. Negative live-charge quantum probes 2 . Zero charged probes 3. Positive live-charge quantum probes and 4 . Positive live-charge nano probes 


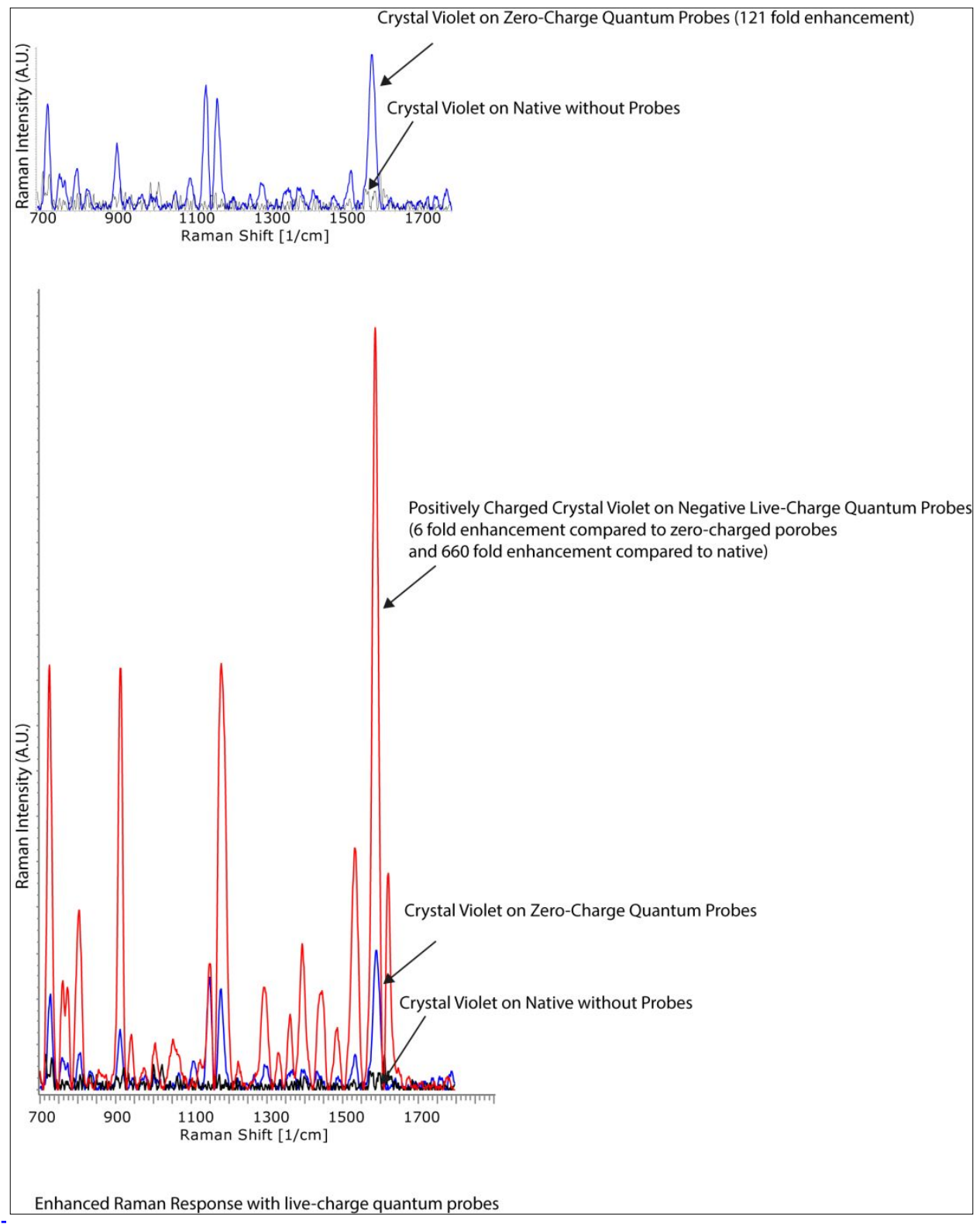

Supporting Fig S9- Raman spectra of Positively charged crystal violet on native without probes, zero-charged quantum probes and negative live-charge quantum probes 


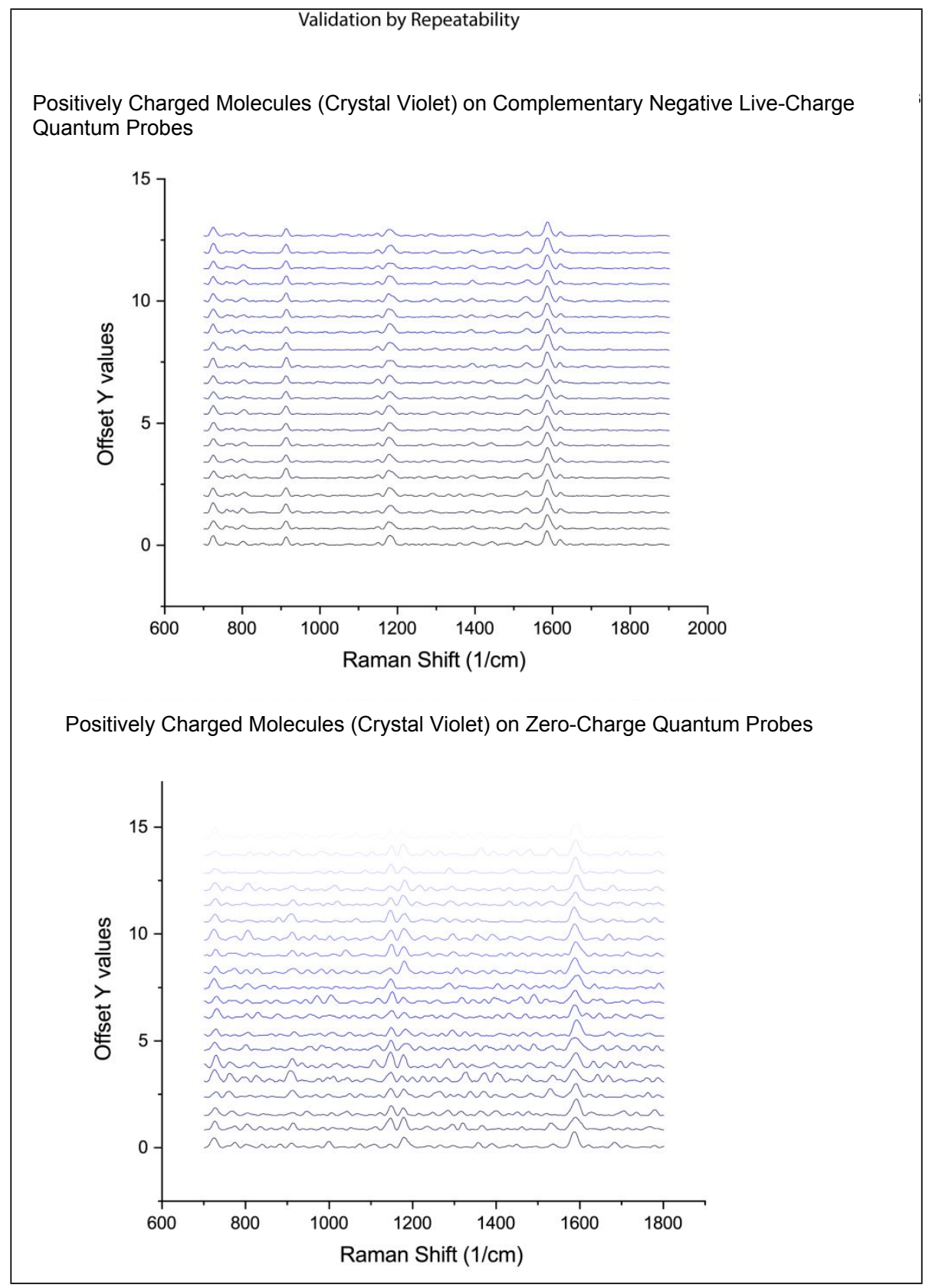

Supporting Fig S10 - Validation by repeatability - Positively Charged Molecules on Negative Live-Charge \& Zero-Charge Probes 


\section{Validation by Reproducibility}

Positively Charged Molecules (Crystal Violet) on Complementary Negative Live-Charge Quantum Probes
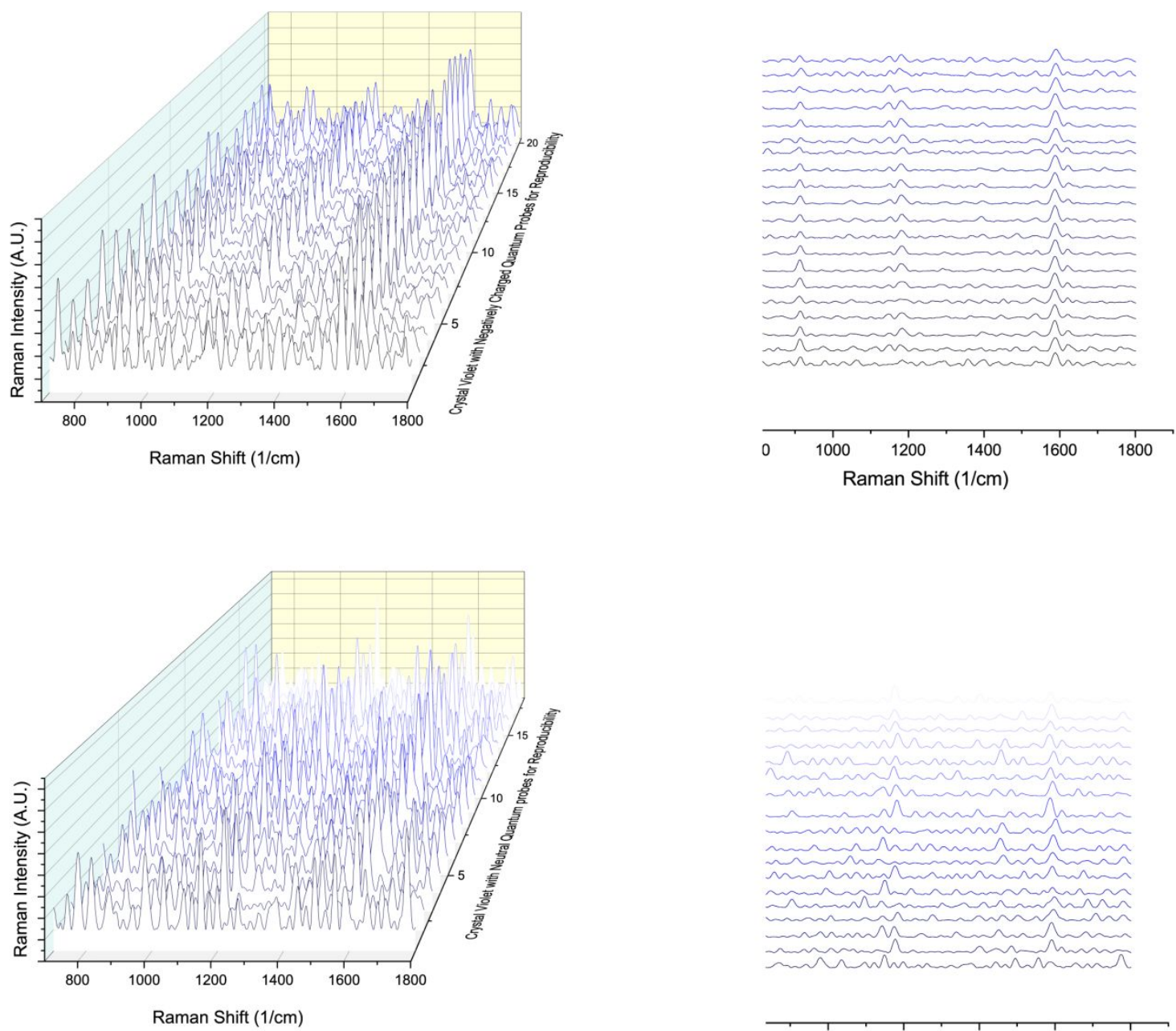

Raman Shift $(1 / \mathrm{cm})$

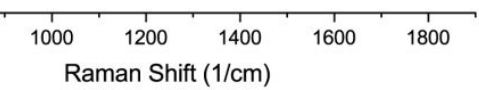

Positively Charged Molecules (Crystal Violet) on Zero-Charge Quantum Probes

Supporting Fig S11 - Validation by reproducibility - Positively Charged Molecules on Negative Live-Charge \& Zero-Charge Quantum Probes 
Validation by Repeatability

Negatively Charged Molecules (Methyl Orange) on Complementary Positive Live-Charge Quantum Probes

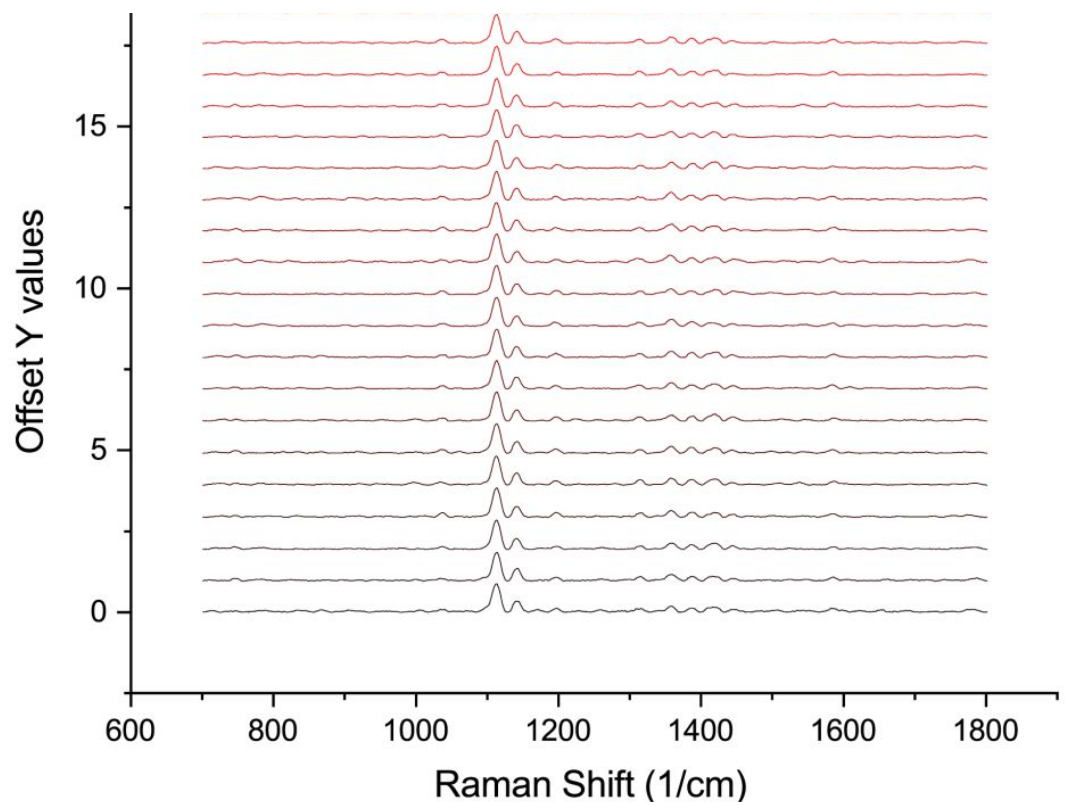

Negatively Charged Molecules (Methyl Orange) on Zero-Charge Quantum Probes

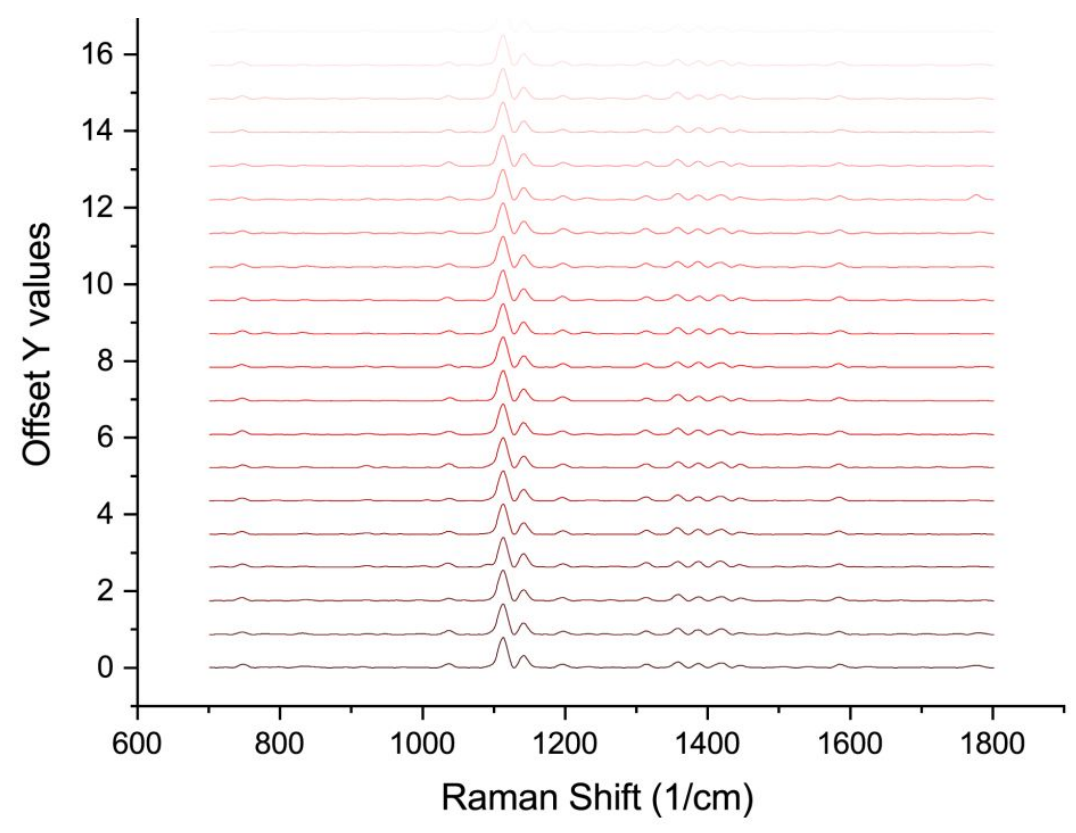

Supporting Fig S12 - Validation by repeatability - Negatively Charged Molecules on Positive Live-Charge \& Zero-Charge Probes 
Positively Charged Molecules (Crystal Violet) on Complementary Negative Live-Charge Quantum Probes
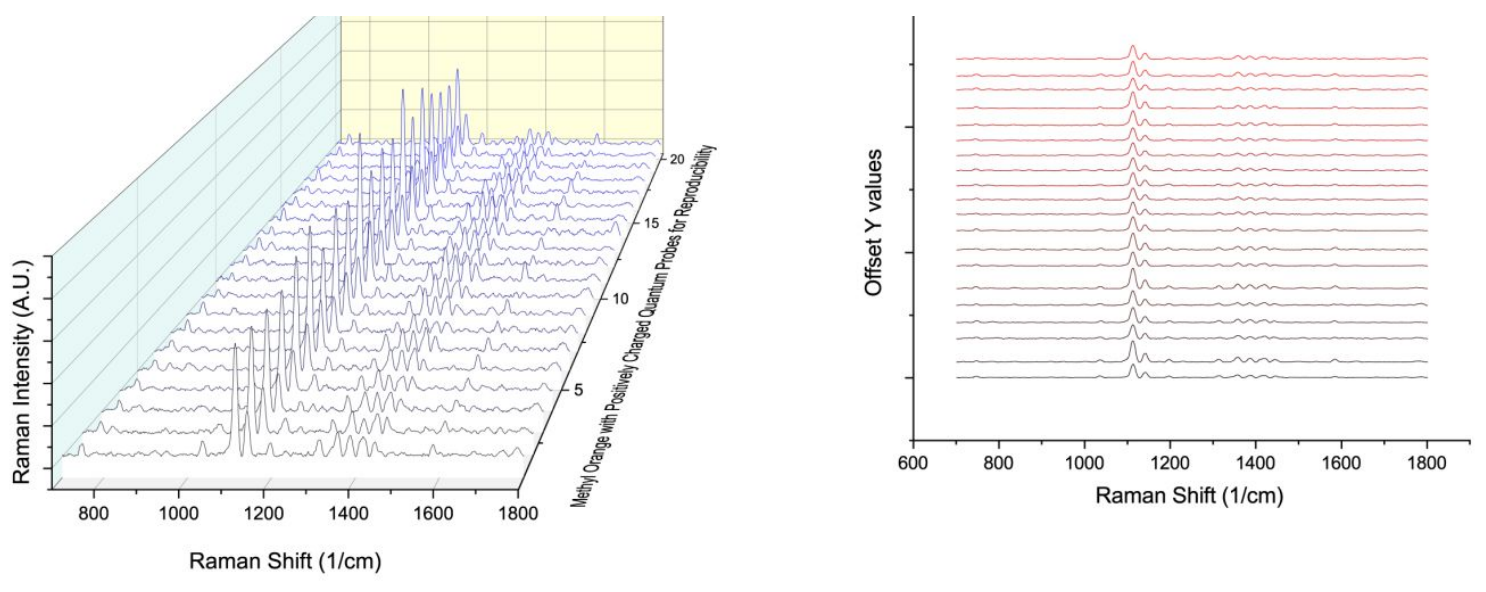

Negatively Charged Molecules (Methyl Orange) on Zero-Charge Quantum Probes
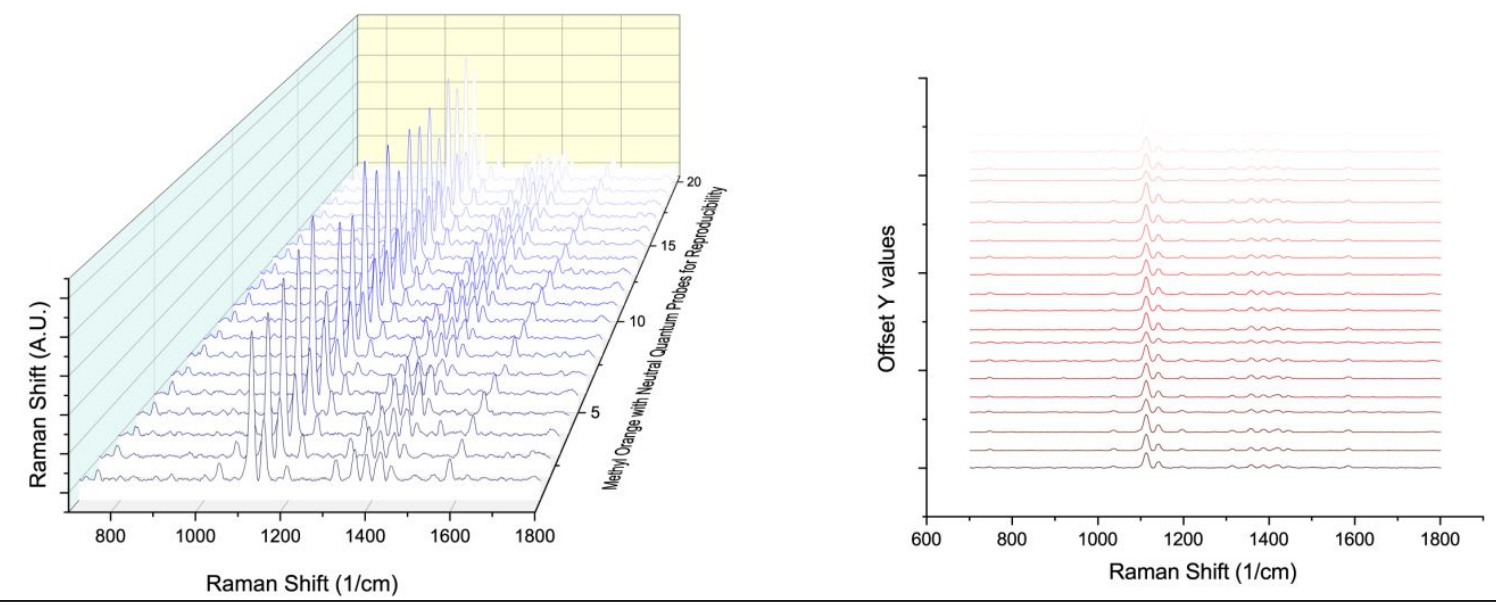

Supporting Fig S13 - Validation by reproducibility - Negatively Charged Molecules on Positive Live-Charge \& Zero-Charge Probes 
Supporting Table 1

\begin{tabular}{|l|l|l|l|}
\hline Type of Probe & $\begin{array}{l}\text { Binding Energies of } \\
\text { Doublet Peaks of XPS, }\end{array}$ & $\begin{array}{l}\text { Binding Energy } \\
\text { Difference (eV) }\end{array}$ \\
& \multicolumn{2}{|l|}{ Zn2P spectra (eV) } & \\
\hline Negative Live-Charge Quantum Probes & 1021 & 1044.09 & 23.09 \\
\hline Zero Charge Quantum Probes & 1020.9 & 1044.02 & 23.12 \\
\hline Positive Live-Charge quantum Probes & 1022.29 & 1045.36 & 23.07 \\
\hline Positive Live-Charge Nano Probes & 1021.03 & 1044.09 & 23.06 \\
\hline
\end{tabular}

We have chosen a semiconductor substrate for the live-charge based Raman enhancement, as semiconductor quantum probes demonstrated dominance of the charge transfer mechanism for SERS response [1]. Also, there is a possibility of optimizing the spatial enclosure of the electronic charge carriers due to the quantum confinement to tune the optical transitions as well as energies of the discrete electronics states of the semiconductors [2]. Also, the reduced size of the probes of the semiconductor material can also demonstrate discrete electronic transitions resembling isolated atoms and molecules [2].

References:

[1] R. Haldavnekar, K. Venkatakrishnan, and B. Tan, "Non Plasmonic Semiconductor Quantum SERS Probe As A Pathway For In Vitro Cancer Detection," Nat. Commun., vol. 9, 2018, doi: 10.1038/s41467-018-05237-x.

[2] D. Gammon, "Electrons in artificial atoms: Semiconductor physics," Nature, vol. 405, no. 6789, pp. 899-900, Jun. 2000, doi: 10.1038/35016189. 